\title{
STUDY OF ELECTRO-PNEUMATIC INTERFACE BASED ON ACOUSTIC LAMINAR TO TURBULENT TRANSITION
}

\author{
G.Belforte, G. Eula, C.Ferraresi, A.Manuello Bertetto and V.Viktorov \\ Department of Mechanics - Politecnico di Torino Technical University \\ C.so Duca degli Abruzzi,24 - 10129 Torino - ITALY \\ (E-mail ferraresi@polito.it)
}

\begin{abstract}
Thanks to the development of piezoelectric materials able to realize high efficiency vibration generators, it is now possible to develop interface devices between low power electric signals and pneumatic signals, based on the phenomenon of laminar to turbulent transition of air jets. Aim of this work is the study of such kind of devices, particularly oriented to the use of electro-acoustic signals. The paper describes a study carried out on a particular experimental device which allowed to identify the influence of various geometric parameters on the transition effect. A campaign of tests has been completed aimed to a dimensional optimisation of the device. The possible utilisation of the device as interface element for control of pneumatic valves has been considered.
\end{abstract}

\section{KEYWORDS}

Electro-pneumatic interface; low electric power interface; laminar-turbulent amplifier.

\section{INTRODUCTION}

The pneumatic systems have had more and more integration with sensors and with control electronic devices. The disadvantage of the electrical commands normally used is the signal's voltage (generally 12 or $24 \mathrm{~V}$ ), the problem being that such a value in some applications is excessive. In addition to this, during their operation these systems absorb a certain amount of power. So there is a need to study the possibility of realising a new kind of pilot stages capable of operating at supply voltages much lower than $12 \mathrm{~V}$, and which will also reduce the level of energy consumption.

Low power control electro-pneumatic valves have been already constructed, using piezoelectric pilot stages [1], but characterised by rather low dynamic behaviour: they are used especially in explosive enviroments because of their intrinsic safety characteristics.

Previous experiences demonstrated that it is possible to achieve very quick and low-power electro-pneumatic pilot stages (around $1 \mathrm{~mW}$ ) by using the laminar/turbulent transition of an air jet excited by a proper device [2]. This work studies the possibility of developing a new kind of very low power electronicpneumatic interfaces, exploiting the transition of a fluidic jet from laminar to turbulent operating condition.
This phenomenon was already used in sixty years to construct fluidic amplifiers [3-7]; it is the basis of the operating principle of laminar-turbulent amplifiers: these are fluidic elements without moving parts, which use fluidodynamics whereby a main supply jet (laminar) is disturbed and made turbulent by the application of a fluidic control signal. As can be seen from the literature [6]-[7], the transition of a jet from laminar to turbulent regime is influenced by many factors such as, for example, speed and viscosity of the fluid, shape and sizes of the cross section of the duct in which the fluid flows. It is well known that an acoustic signal can be used to perturbate a laminar jet and made it turbulent [8][9].

In order to optimise the efficiency of the amplifier, it is then necessary to deepen the knowledge of the behaviour of the jet under the effect of an acoustic signal. The aim of this work was to develop a laminar/turbulent amplifier suitable to be used as a very low power pilot interface for pneumatic valves. The amplifier should be able to generate a proper level of recovered pressure in absence of the control signal, and should reduce the recovered pressure to as small as possible level when activated. It is known that the transition of a jet from laminar to turbulent condition depends on the frequency of the acoustic control signal. So it is possible to individuate for a jet some "critical 
frequencies" at which the laminar/turbulent transition happens more easily.

A simple way to generate a proper acoustic signal is then to use a common loudspeaker, usually characterised by a flat frequency response. However this solution is not very convenient as regards the energy consumption. More attractive could be the use of a piezoelectric disc: in fact it can generate an acoustic signal with very low-power excitation. The only drawback is that this device has a non-flat frequency response, that shows typical resonant frequencies. When a piezoelectric disc is used as acoustic signal generator, its efficiency is maximised when it is excited at such resonant frequencies.

Therefore, in order to maximise the efficiency of such an electro-pneumatic interface, it is important that the resonant frequencies of the piezoelectric disc closely match the critical frequencies of the jet. To investigate the influence of the geometry and of the characteristics of the control signal on the behaviour of the amplifier, a proper experimental device was made in which the most relevant geometric parameters could be varied. A first optimisation of the amplifier geometry has been carried out by using a pneumatic control signal. Then, an acoustic characterisation of the jet has been carried out using the signal generated by a loudspeaker with flat frequency response, in order to individuate the critical frequencies of the jet. Finally, the influence of the geometrical configuration on the amplifier efficiency has been investigated when the acoustic control signal was generated by a piezoelectric disc.

\section{EXPERIMENTAL DEVICE DESCRIPTION}

The experimental device is shown in figure 1a. It is made of a body inside of which two nozzles have been positioned, 1 and 2, flanked on a chamber 3 that is open on two sides in order to permit the discharge of air. The jet, coming from the supply nozzle 1 is, in the absence of the control signal, laminar and is gathered at the exit nozzle 2 with a certain retrieval level. The presence of the control signal provokes turbulent transition and a fall of the exit signal. The efficiency of the device is improved the more the signal tends towards zero when the control signal is applied.

As suggested in previous studies on laminar-turbulent amplifiers [9], an auxiliary device has been placed between emitter and receiver to improve the device's performance. This element, known as the diffuser, is traditionally made up of an insert with an internal conical surface. In this work a more simple element has been adopted, made up of two coaxial parts ( 1 and 2 in figure $1 \mathrm{~b}$ ) with axial symmetry, having central holes with different diameters $d_{1}$ and $D_{1}$. The axial length of the diffuser was equal to $4 \mathrm{~mm}$. The prototype (fig. la) has the following features: the dimension of the space 3 that exists between the nozzles does not disturb the jet and presents sufficiently large discharge ways for a rapid outflow of air not recovered by the receiving nozzle 2 ; it can send, via a purpose made conduit, a pneumatic control signal supplied separately by the emitting nozzle 1; it is also possible to send an acoustic control signal, as the space 3 between the two nozzles is open and sound waves can hit the jet and generate the laminar to turbulent transition; the shape of the above-mentioned chamber 3 allows for the insertion, at variable distances, of a diffuser between the nozzles 1 and 2; the device guarantees the coaxiality of the two nozzles 1 and 2 regardless of the distance at which they have been placed.

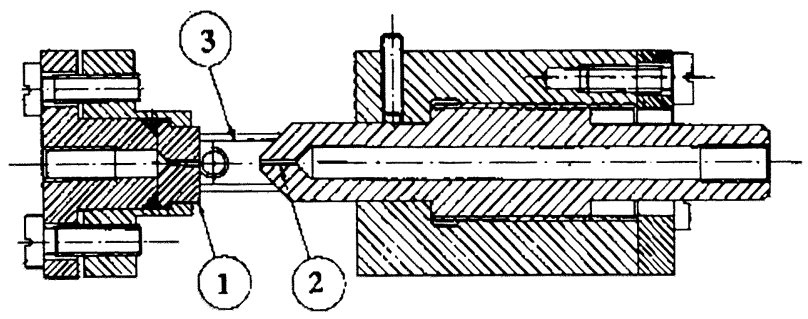

a)

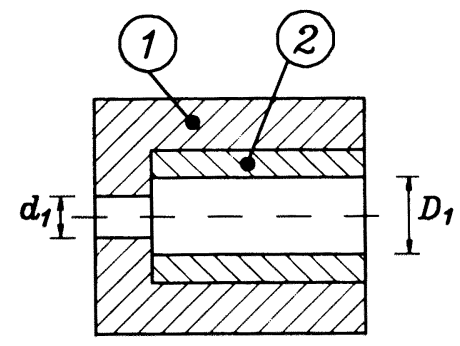

b)

Figure 1 a) Laminar-turbulent amplifier prototype b) Diffuser element

\section{TEST METHODOLOGY}

The experimental tests were made using different circuits, depending on the aims that had to be pursued. The analysis was made by varying the geometry, the supply pressure, the type of control signal and its intensity. From a geometrical point of view the following parameters were varied: the distance $\mathrm{L}$ between the nozzles, the diameter $d$ of the emitting and receiving nozzles. In all tests here presented emitting and receiving nozzles have the same diameter $d$. The supply flow rate was measured to calculate the Reynolds number, defined as: 


$$
\operatorname{Re}=\frac{\rho v d}{\mu}
$$

where $\mathrm{v}$ is the mean velocity of the fluid in the emitting nozzle; $d$ is the characteristic geometrical dimension (emitting nozzle diameter); $\rho$ is the density of the fluid; $\mu$ is the viscosity of the fluid.

The first control signal used was of pneumatic type with values for the control pressure variable from 0 to $1000 \mathrm{~Pa}$; the second was acoustic, generated firstly by a loudspeaker (with variable frequencies from 2000 to $22000 \mathrm{~Hz}$ ), and then by a piezoelectric disc. In general the supply pressures were set between 1500 and 3000 $\mathrm{Pa}$.

\section{TEST WITH PNEUMATIC CONTROL}

Figure 2 shows the test bench used to analyse the behaviour of the prototype when a pneumatic control was used. In this scheme the prototype is represented by two nozzles 1 and 2, while nozzle 3 generates the control signal.

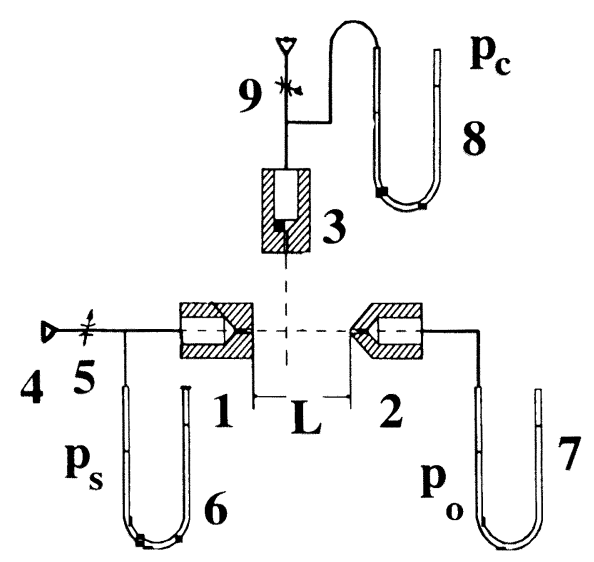

Figure 2 Experimental set-up used with a pneumatic control.

The working fluid (air) enters the system via supply 4, and resistance 5 enables the regulation of supply pressure. The manometers 6,7 and 8 (resolution about $10 \mathrm{~Pa}$ ) measure the supply pressure $\mathrm{p}_{\mathrm{s}}$, output pressure $p_{o}$ and control pressure $p_{c}$ respectively. The latter is regulated using resistance 9 . The influence of distance $\mathrm{L}$ between the nozzles was studied by varying it in a field in which it was possible to observe the phenomenon of the laminar to turbulent transition. This was studied also for different positions of the receiving nozzle 2 along its own axis. The minimum distance $\mathrm{L}$ used was equal to $8 \mathrm{~mm}$, the maximum was $14 \mathrm{~mm}$. Values of $\mathrm{L}$ have been measured using a micrometer with resolution about $0.01 \mathrm{~mm}$. In order to eliminate a certain residual pressure at the exit of the system, the diffuser was placed between the two nozzles. The tests were carried out with different values of distance $\mathrm{X}$ between diffuser and emitting nozzle, varied from 0 to $5 \mathrm{~mm}$. The best results were obtained with a diffuser placed at a distance $X=4-5 \mathrm{~mm}$ from the emitting nozzle.

Figure 3 shows four curves indicating the output pressure $p_{o}$ versus the control pressure $p_{c}$ and obtained with four different geometrical configurations. In particular, two configurations considered the presence of the diffuser between the nozzles, while the remaining two were without diffuser. Table 1 reports the details of the four configurations tested. In all these conditions the supply pressure $\mathrm{p}_{\mathrm{s}}$ was equal to $2400 \mathrm{~Pa}$ and the distance $\mathrm{X}$ between diffuser and emitting nozzle was $5 \mathrm{~mm}$.

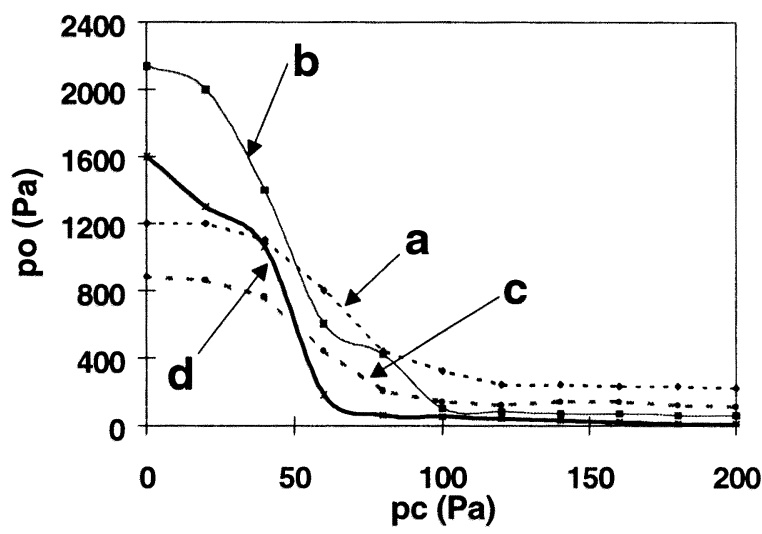

Figure 3 Output pressure with pneumatically controlled device

It is evident that the diffuser improves the efficiency of the amplifier: the two corresponding curves ( $b$ and d) show a relatively high recovered pressure in the absence of control signal, while for control pressure above $90 \mathrm{~Pa}$ the output has a low residual value (around $50 \mathrm{~Pa}$ ). On the contrary, the curves a and c (amplifier without diffuser) show a lower recovered pressure and a higher residual pressure. In figure 3 experimental data are the mean value of subsequent measures, which maximum dispersion, due to the transition jet, is about $5-10 \%$.

Figure 4 shows the results obtained with comparative tests carried out for the three different diameter of nozzles, with the same value of $L=10 \mathrm{~mm}$ and $X=5$ $\mathrm{mm}$. In all tests the diffuser was used. The details of the three configurations tested are shown in Table 2 . 
Table 1 Configuration for test with pneumatic control

\begin{tabular}{|c|c|c|c|c|c|}
\hline Config. & $\begin{array}{c}\mathrm{d} \\
(\mathrm{mm})\end{array}$ & $\begin{array}{c}\mathrm{L} \\
(\mathrm{mm})\end{array}$ & diffuser & $\begin{array}{c}\mathrm{d}_{1} \\
(\mathbf{m m})\end{array}$ & $\begin{array}{c}\mathrm{D}_{1} \\
(\mathbf{m m})\end{array}$ \\
\hline $\mathrm{a}$ & 0.5 & 10 & NO & none & none \\
b & 0.5 & 10 & YES & 0.7 & 1 \\
\hline c & 0.5 & 12 & NO & none & none \\
d & 0.5 & 12 & YES & 0.7 & 1 \\
\hline
\end{tabular}

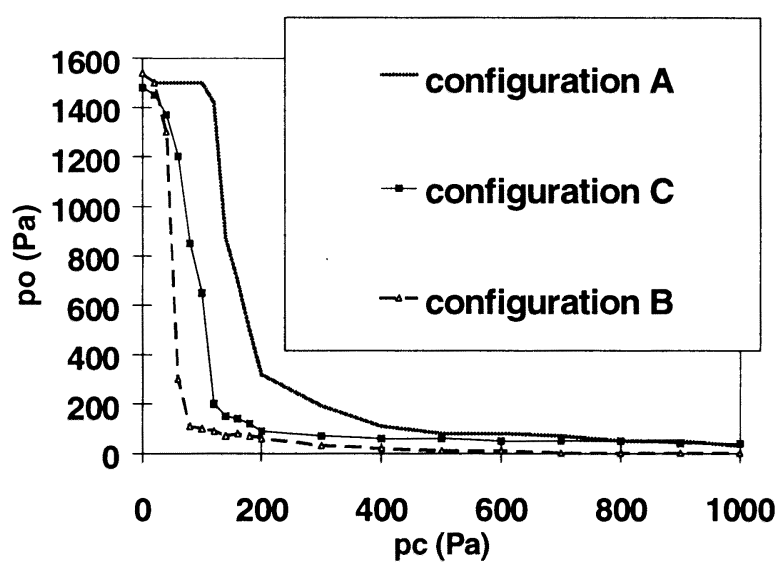

Figure 4 Comparative tests with different diameter nozzles.

Table 2 Configuration tested at different nozzle diameters

\begin{tabular}{|c|c|c|c|c|}
\hline Configuration & $\begin{array}{c}\mathbf{d} \\
(\mathbf{m m})\end{array}$ & $\begin{array}{c}\mathbf{L} \\
(\mathbf{m m})\end{array}$ & $\begin{array}{c}\mathbf{d}_{\mathbf{1}} \\
(\mathbf{m m})\end{array}$ & $\begin{array}{c}\mathbf{D}_{1} \\
(\mathbf{m m})\end{array}$ \\
\hline A & 0.4 & 10 & 0.6 & 1.2 \\
\hline B & 0.5 & 12 & 0.7 & 1.5 \\
\hline C & 0.7 & 14 & 1.0 & 2.1 \\
\hline
\end{tabular}

The supply pressure $p_{s}$ was equal to $2400 ; 2000$ and $1600 \mathrm{~Pa}$ for configurations $\mathrm{A}, \mathrm{B}$ and $\mathrm{C}$ respectively. Reynolds number was about 1330 with $\mathrm{d}=0.4 \mathrm{~mm}$, 1570 with $d=0.5 \mathrm{~mm}$ and 1500 with $\mathrm{d}=0.7 \mathrm{~mm}$. It is clear that the best results have been obtained with $d=$ $0.5 \mathrm{~mm}$ (configuration $\mathrm{B}$ ).

\section{ACOUSTIC CONTROL BY LOUDSPEAKER}

The test scheme used is illustrated in figure 5. In this case the command signal was constituted by sound waves generated with the loudspeaker LO, excited by the signal generator $G$ and by the amplifier $A$. The analyser SA reads the signal of the generator $G$ and of the microphone $\mathrm{M}$, amplified by the unit $\mathrm{P}$.

These tests established how $p_{o}$ varied versus the acoustic signal generated by the loudspeaker. In particular the working frequency of the latter was varied between 2000 and $22000 \mathrm{~Hz}$, while the amplitude of the loudspeaker signal was maintained constant. By varying the frequency of the loudspeaker signal, it was established for which values this produces the laminar to turbulent transition of the jet and therefore a notable reduction of $\mathrm{p}_{\mathrm{o}}$.

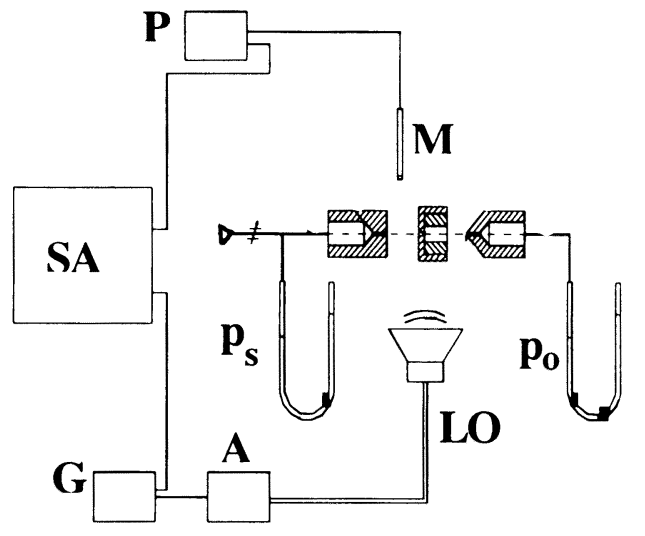

Figure 5 Set-up used with non-pneumatic control

A lot of tests with different $\mathrm{d}$ and $\mathrm{L}$ values have been made and the influence of both these parameters has been seen on the critical frequency at which there is the transition of the jet from the laminar to the turbulent regime. For example in Figure 6 three curves, referred to the three configuration explained in Table 2 , are shown. In this case the supply pressure $\mathrm{p}_{\mathrm{s}}$ was equal to 2400;2000 and $1600 \mathrm{~Pa}$ for configurations A,B and C respectively. In each test the acoustic signal intensity was maintained equal to $110 \mathrm{~dB}$. For each of the three configurations tested, it is possible to individuate a first critical frequency, corresponding to the following approximate values: $6200 \mathrm{~Hz}$ for configuration A; 5300 $\mathrm{Hz}$ for configuration $\mathrm{B} ; 4500 \mathrm{~Hz}$ for configuration $\mathrm{C}$.

Figure 7 shows a comparison between two characteristics obtained with the same diameter of nozzles, $\mathrm{d}=0.5 \mathrm{~mm}$, placed at two different distances, $\mathrm{L}$ $=10 \mathrm{~mm}$ and $\mathrm{L}=11 \mathrm{~mm}$. By analysing these graphs, one can notice that, for the same diameter of nozzles $d$, the value of distance $\mathrm{L}$ influences significantly the value of the first critical frequency of the jet. In particular, a greater $\mathrm{L}$ determines a lower critical frequency. These results show clearly that it is possible, by varying the main geometrical parameters of the 
amplifier, to set the resonant frequency to a desired value.

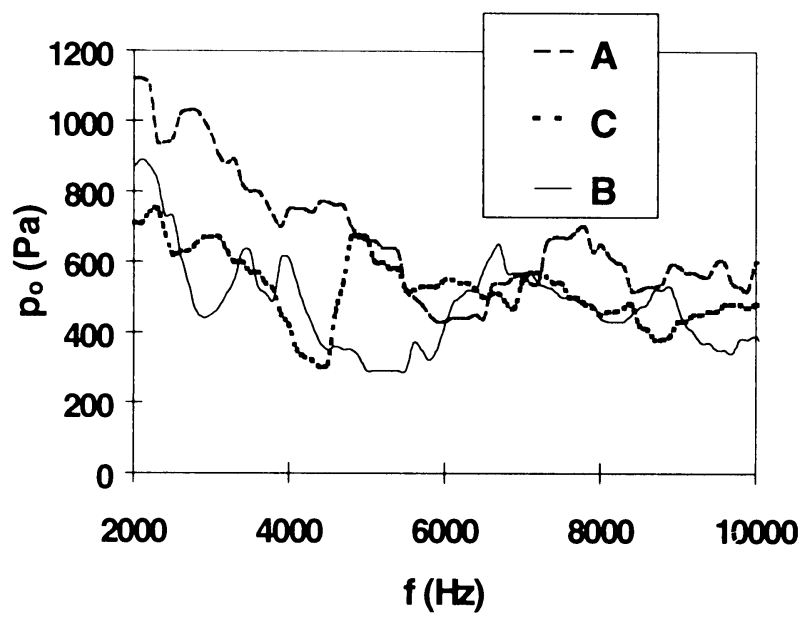

Figure 6 Output pressure of the amplifier versus the frequency of acoustic command

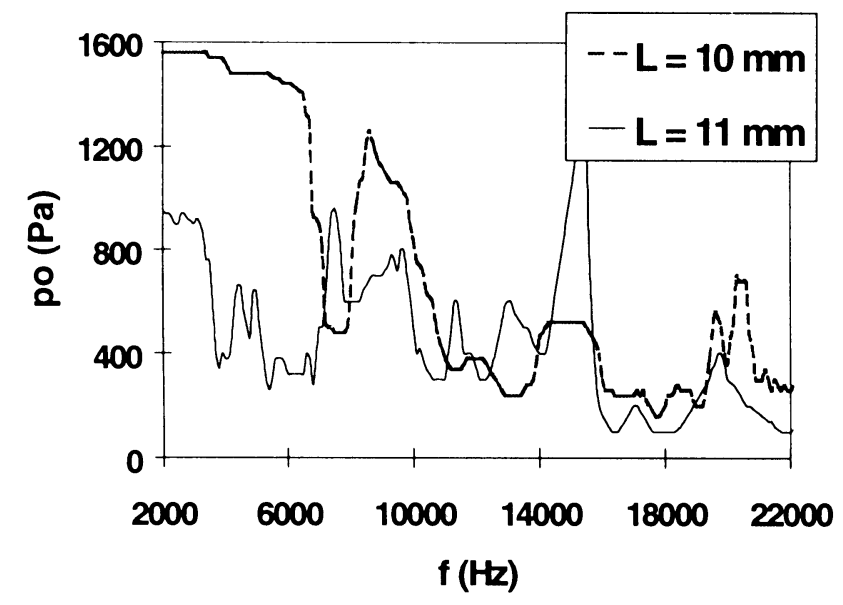

Figure 7 Characteristic $p_{o}$ versus frequency

\section{ACOUSTIC CONTROL BY PIEZOELECTRIC DISC}

The test bench is again shown in figure 5, where in this case the acoustic control signal was generated by a proper element (LO) consisting of a piezoelectric disc deformed under the effect of an alternate electrical signal. Two piezoelectric discs, with diameters $D$ equal to 27 and $20 \mathrm{~mm}$, have been considered. First of all, the frequency response of the discs have been obtained by exciting them with a supply voltage varying from 2000 to $20000 \mathrm{~Hz}$ and acquiring the acoustic signal by the microphone $\mathrm{M}$.
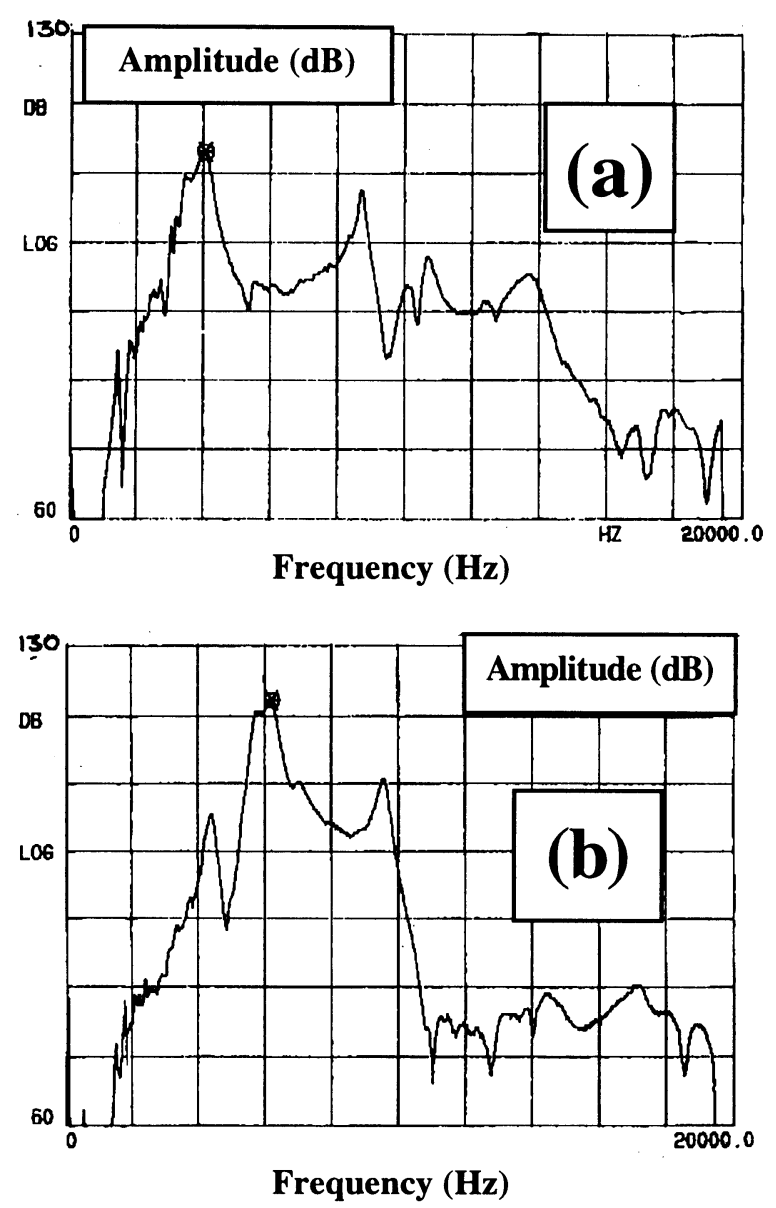

Figure 8 Frequency response of piezoelectric discs

In both cases the supply voltage of the piezoelectric element was equal to $2.5 \mathrm{~V}$; the distance between piezoelectric disc and microphone was equal to $10 \mathrm{~mm}$. Figure $8 \mathrm{a}$ shows the frequency response of the disc with $\mathrm{D}=27 \mathrm{~mm}$. It presents a first resonant frequency at $\mathrm{f}_{\mathrm{r}}=$ $4025 \mathrm{~Hz}$, with a peak of $114 \mathrm{~dB}$. Figure $8 \mathrm{~b}$ illustrates the frequency response of the disc with $\mathrm{D}=20 \mathrm{~mm}$. Its first resonant frequency is at $f_{r}=6000 \mathrm{~Hz}$, with a peak of $123 \mathrm{~dB}$. From the characteristics of the piezoelectric discs one can notice that the disc with $\mathrm{D}=20 \mathrm{~mm}$ has its first resonance close to the critical frequency of the air jet when the geometry of the experimental device is configured as for the case shown by the characteristic in figure $7(\mathrm{~L}=11 \mathrm{~mm})$. Therefore, tests on the laminar-turbulent amplifier were made, in which the acoustic signal was generated by the piezoelectric disc of $\mathrm{D}=20 \mathrm{~mm}$ excited at $6000 \mathrm{~Hz}$ and the device was configured with nozzles of diameter $d=0.5 \mathrm{~mm}$, diffuser with $d_{1}=0.7 \mathrm{~mm}$ and $D_{1}=1.5 \mathrm{~mm}$, placed at $X=5 \mathrm{~mm}$ from the emitting nozzle. The tests were repeated for different supply pressures and with distance between nozzles $\mathrm{L}=10$ and $11 \mathrm{~mm}$. The 
results obtained are presented in figure 9, where the value of the output pressure pressure is drawn versus the voltage input to the piezoelectric disc. It is clear that the geometry corresponding to $\mathrm{L}=11 \mathrm{~mm}$ gives a better behaviour of the system. In fact in these conditions a variation of very few Volts $(0-0.6 \mathrm{~V})$ produces a notable variation of $\mathrm{p}_{\mathrm{o}}$ (about $1400 \mathrm{~Pa}$ ) with low residual pressure level.

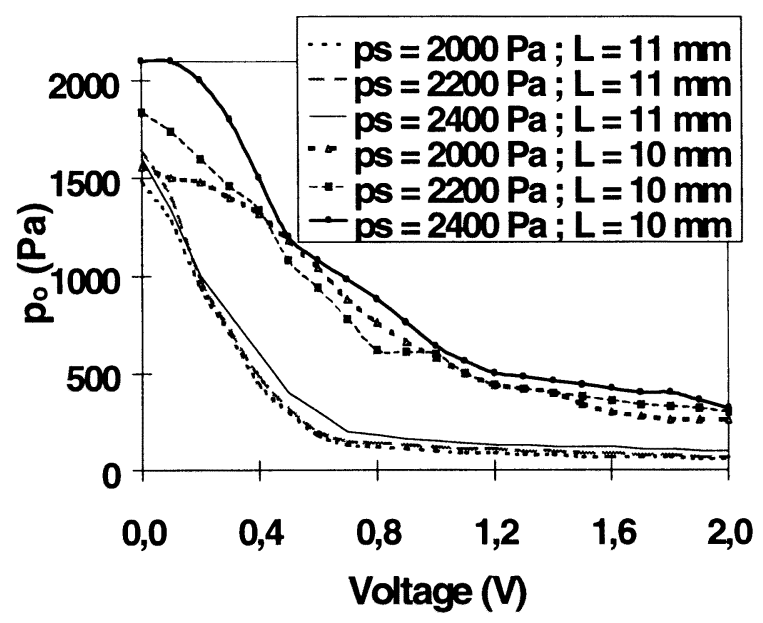

Figure 9 Output pressure obtained with different voltages of piezoelectric control

This confirms that the closer the working frequency of the piezoelectric element gets to the resonant frequency of the jet, the more the jet becomes disturbed and the output pressure drops. In figure 9 the experimental data are the mean value of subsequent measures, which maximum dispersion, due to the transition jet, is about $5-10 \%$.

\section{CONCLUSIONS}

This work allowed to clarify some important aspects that determine the behaviour of a laminar-turbulent amplifier controlled by an acoustic signal. In particular:

- it is possible to define an "acoustical characteristic" of the jet, i.e. to individuate some critical frequencies at which the jet can be made turbulent more easily;

- these critical frequencies depend closely on the geometry of the amplifier;

- it is possible to realise an amplifier with a given critical frequency by varying properly some geometrical parameters, in particular diameter of nozzles and distance between them;

- the amplifier's efficiency was improved by the insertion between nozzles of a diffuser element with very simple geometry;
- a particularly efficient amplifier can be realised by using as acoustic generator a piezoelectric disc, excited at its resonant frequency corresponding to a critical frequency of the jet.

In this way it is possible to realise a very low power electro-pneumatic interface, that could be used as a pilot stage for pneumatic valves.

\section{ACKNOWLEDGEMENTS}

We would like to thank Eng. Marco Bonfadini for his collaboration during the tests.

The research was financed by MURST (Italian Ministry for University and Research).

\section{REFERENCES}

1. Finn, A.M., Tavrow, L.S., Brooks, R.A., Ehrlich, D.J., Udayakumar, K.R., Cross, L.E., Piezoelectric Micromotors for microrobots, J.of Microelectromechanical Systems, 1992, 1-1, pp.4451.

2. Belforte G., Ferraresi C., Eula G., Study of a new electro-pneumatic interface, 5th Triennal Symp. on Fluid Control, Measur. and Visual., Flucome'97, Japan, 1997, 2, pp.551-556.

3. Auger R.N., The Turbulence amplifier in control systems, Fluid. Ampl. Symp., HDL, Whashington, USA, 1964.

4. Verhelst H.A.M., On the design, characteristics and production of turbulence amplifiers, 2nd Cranfield Fluid. Conf., B.H.R.A., Cranfield, UK, 1967, paper F2.

5. V.Gradetsky, V.Dimitriev, M.Sons, Some design problems of the fluidic digital elements and devices, 3rd Cranfield Fluid. Conf., B.H.R.A., Cranfield, UK, 1968, paper T1.

6. Rolland J., Digital step-pipe amplifier application in gas distribution control, 3rd Cranfield Fluid. Conf., B.H.R.A., Cranfield, UK, 1968, paper B7.

7. Ferhmann R., Matko J., Shubert W., Shulze W. and Tittle H., Planar turbulence amplifier in stack contruction and industrial application of this technique, 4th Cranfield Fluid. Conf., B.H.R.A., Cranfield, UK, 1970, paper A2.

8. Bramley H.C., Hu F.Q.and J.M.Watson, Optical control, for a pneumatic actuator, European J. of Fluid Power, Noregren Martonair Limited, 5-4, 1993, pp.16-19.

9. Belforte G., Eula G., Ferraresi C. and Viktorov V., Development of a new electronic-pneumatic control for pneumatic systems, Mechatronics 98, Sweden, 1998, pp.55-60. 